\title{
Studies of Polyelectrolyte Solutions V. Effects of Counterion Binding by Polyions of Varying Charge Density and Constant Degree of Polymerization
}

\author{
Eckhard NORDMEIER* \\ Department of Physical Chemistry, University of Osnabrück \\ 49069 Osnabrück, FRG
}

(Received August 19, 1993)

\begin{abstract}
The effect of the charge density on the behaviour of polyelectrolytes in contact with counterions was studied by activity measurements, Donnan dialysis, dye-spectrophotometry, and potentiometric titration. The polyelectrolytes investigated were poly(acrylic acid), i.e., PAA, and acrylic acid/acrylamide copolymers, i.e., PAM-y, where $y$ is the degree of hydrolysis. This gave charge density parameters, $\xi$, which range from 0.12 to 2.88 . Activity coefficients were determined for $\mathrm{Na}^{+}$and $\mathrm{K}^{+}$ions at $T=25^{\circ} \mathrm{C}$. The results were compared with those predicted from the theories of Manning and of Iwasa. Above the critical value, $\xi_{c}=1$, agreement with theory is satisfactory, but for $\xi<1$ there are significant deviations. The probable reason is change in the polyion flexibility. Binding isotherms for the univalent counterions, $\mathrm{Na}^{+}$and $\mathrm{K}^{+}$, were determined by utilizing the dialysis technique. It is found that the degrees of binding, $\theta_{\mathrm{Na}}$ and $\theta_{\mathrm{K}}$, decrease with decreasing $\xi$ as predicted from theory. For $\xi<1$, the experimental error in $\theta_{\mathbf{N a}}$ and $\theta_{\mathbf{K}}$ becomes very large $(>20 \%)$, but within this error no counterion binding is observed. The binding of the divalent counterion $\mathrm{Mg}^{2+}$ was examined by a dye spectrophotometric method, where $\mathrm{Na}^{+}$or $\mathrm{K}^{+}$ ions were added in excess. The results were analyzed within Manning's two-variable theory. For $\xi>1$, the binding isotherms are in reasonable agreement with theory. For $\xi<1$, the Manning theory tends to under-estimate the degree of $\mathrm{Mg}^{2+}$ binding, $\theta_{\mathbf{M g}}$. Interestingly, $\theta_{\mathbf{M g}}$ is not zero at $\xi=0.28$, suggesting that for divalent ions counterion binding occurs above $\xi_{\mathrm{c}}=0.5$ as well as below. Potentiometric titration shows that there is some polyion folding for PAM- $y$. The degree of folding increases with decreasing $y$. This leads to increase of the effective polyion charge, so that $\xi$ should be replaced by $\xi_{\text {eff }}$. It is observed that there is a small binding specificity for $\mathrm{Na}^{+}$over $\mathrm{K}^{+}$at large $\xi$. This vanishes when $\xi$ becomes small. The reason is not clear yet. It may be due to increase in the content of helical segments of PAM- $y$ as $y$ decreases.
\end{abstract}

KEY WORDS Poly(acrylic acid/acrylamide) / Activity Coefficients /

Counterion Binding / Potentiometric Titration /

A number of attempts ${ }^{1-3}$ have been made to theoretically explain the physicochemical properties of polyelectrolyte solutions. However, the theories have not proven completely satisfactory. This indicates that our understanding of the subject is still incomplete. Perhaps, the greatest theoretical problems arise from the high charge density on the polyion which makes application of the simple DebyeHückel theory unacceptable. To overcome this difficulty the concept of counterion binding ${ }^{4}$ has been found of great utility.

However, the definition of counterion binding in physical terms is still ambiguous. It can be divided into two types. Some of the counterions are attracted to a volume $V_{\mathbf{P}}$ of

\footnotetext{
* Correspondence should be addressed to: E. Nordmeier, Platanenallee 9, 49152 Bad Essen, Germány.
} 
the polyion domain through electrostatic interactions. Although these ions are localized to some extent, they are mobile. This type of binding is referred to as diffuse or territorial binding. Other counterions are attracted to specific groups of the polyion through formation of ion pairs. These counterions are completely localized and become part of the polyion backbone. This type of binding is referred to as site or localized binding. In general, overall counterion binding is a combination of both types, possibly with one type predominant.

Several thermodynamic and electric transport properties have been employed ${ }^{5-7}$ to examine the type and extent of counterion binding. The results obtained, however, differ significantly. This is because the experimental techniques used are diversely sensitive for the two types of binding.

The present study reports counterion binding experiments on polyelectrolytes of varying charge density and constant degree of polymerization. Very little work $^{8-10}$ has been done in this field, although it is well recognized that the charge density is the most important parameter affecting the solution properties of a polyion.

We focus our attention to acrylic acid/acrylamide copolymers, PAM-y. Their charge density can be varied by changing the ratio, $y$, of acrylic acid to acrylamide to any desired extent without altering the degree of polymerization. This is readily achieved by hydrolyzing nonionic polyacrylamide with $\mathrm{NaOH}$.

The paper is organized as follows. First, we consider $\mathrm{Na}^{+}$and $\mathrm{K}^{+}$activity coefficients of poly(acrylic acid) called PAA and of PAM- $y$. The charge density, $\xi$, covers the range from 0.12 to 2.88 . The results are compared with predictions of the theories of Manning ${ }^{11}$ and Iwasa. ${ }^{12}$ We query whether there is the possibility of selective counterion binding caused by the PAM-y acrylamide groups. Second, we examine the counterion binding of $\mathrm{Na}^{+}, \mathrm{K}^{+}$, and $\mathrm{Mg}^{2+}$ ions, where for $\mathrm{Mg}^{2+}$ the
$\mathrm{Na}^{+}$and $\mathrm{K}^{+}$ions are added in excess. Theory ${ }^{11}$ predicts that when $\xi$ is larger than $\xi_{\mathrm{c}}$, where $\xi_{c}$ is the critical charge density, counterions condense (bind) onto the polyion until $\xi$ is equal to $\xi_{c}$. The remaining counterions are free, i.e., they are localized in the polyion DebyeHückel atmosphere. Theoretical justification of Manning's condensation theory was given by LeBret and Zimm. ${ }^{13}$ Experimental evidence exists only for $\xi>\xi_{\mathrm{c}}{ }^{14,15}$ Thus, we query what is with $\xi<\xi_{\mathrm{c}}$. Third, we explore the possibility of polyion folding by potentiometric titration. Such a conformation transition would change the actual value of $\xi$ so that $\xi$ should be replaced by $\xi_{\text {eff }}$.

Altogether this paper should contribute to a deeper understanding of polyion behaviour and help to elucidate biological phenomena such as ion carriers or ion channels.

\section{EXPERIMENTAL}

\section{Materials}

Poly(acrylamide), PAM, was purchased from Stockhausen. It has a molecular mass, $M_{w}$, of $1.75 \times 10^{6} \mathrm{~g} \mathrm{~mol}^{-1}$, which was determined by small angle static light scattering in $0.1 \mathrm{M} \mathrm{NaCl}$ at $T=25^{\circ} \mathrm{C}$. The dye Eriochrome Black $\mathrm{T}$ (EBT) and the poly(acrylic acid), PAA, were from Aldrich. The molar mass of PAA is $2.1 \times 10^{6} \mathrm{~g} \mathrm{~mol}^{-1}$. The salts used are $\mathrm{NaNO}_{3}, \mathrm{KNO}_{3}$, and $\mathrm{Mg}\left(\mathrm{NO}_{3}\right)_{2}$. They were of analytical grade (from Merck).

\section{Copolymer Preparation}

The acrylic acid/acrylamide copolymers were prepared by hydrolyzing poly(acrylamide) under alkaline conditions. In detail, 1 liter of solution, containing $1 \%$ (by mass) of PAM was kept in a well stirred reaction vessel. The temperature of this vessel was controlled at $60^{\circ} \mathrm{C}$ and $\mathrm{NaOH}$ was added to give a $2 \mathrm{M}$ $\mathrm{NaOH}$ solution. Following hydrolysis at different time intervals between 0.1 and $150 \mathrm{~h}$, samples were taken from the reaction mixture. These were acidified to $\mathrm{pH} \approx 1$ and then purified 
Table I. Several properties of the polyelectrolytes studied

\begin{tabular}{lrrr}
\hline Sample & $y / \%$ & $b / \AA$ & $\xi$ \\
\hline PAM-4 & 4 & 60.0 & 0.12 \\
PAM-10 & 10 & 25.7 & 0.28 \\
PAM-16 & 16 & 16.0 & 0.45 \\
PAM-30 & 30 & 8.4 & 0.86 \\
PAM-42 & 42 & 5.9 & 1.22 \\
PAM-68 & 68 & 3.7 & 1.96 \\
PAA & 100 & 2.5 & 2.88 \\
\hline
\end{tabular}

by exhaustive dialysis against doubly distilled water. After that, aliquots of the purified copolymers were freeze dried and elemental analysis was performed to determine the degrees of hydrolysis. Finally, the polyacids were converted to their $\mathrm{Na}$ or $\mathrm{K}$ salts by neutralization with $\mathrm{NaOH}$ or $\mathrm{KOH}$ and stored in a cold room at $5^{\circ} \mathrm{C}$.

To distinguish between the different acryl acid/acrylamide copolymers prepared, we label them PAM- $y$, where $y$ is the degree of hydrolysis, i.e., the acrylic content per copolymer in mole percent. Table I lists the various structural parameters used. $b$ is the average spacing between two carboxylate groups on the polyion and $\xi$ is the charge density parameter, so that

$$
\xi=e^{2} /\left(4 \pi \varepsilon_{0} \varepsilon k_{\mathrm{B}} T b\right)
$$

Here, $e$ is the elemental charge, $\varepsilon$ is the dielectric constant of the solvent, $k_{\mathrm{B}}$ is the Boltzmann constant, and $T$ is the temperature in Kelvin. The maximal degree of hydrolysis achieved is nearly $70 \mathrm{~mol} \%$. This value is in good accord with the limiting values reported by other workers. ${ }^{16,17}$ An explanation is that at high $y$ the Coulombic repulsion of $\mathrm{OH}^{-}$by two $\mathrm{COO}^{-}$-groups is so strong that hydrolysis is no longer possible. This is supported by the fact that the acrylic acid singlets and doublets are distributed randomly along a PAM- $y$ chain.

\section{Activity Measurements}

Activity coefficients of $\mathrm{Na}^{+}$and $\mathrm{K}^{+}$ions were determined with a Metrohm cationic electrode at $25^{\circ} \mathrm{C}$. The EMF-analysis was carried out as described by Gekko et al. ${ }^{18}$.

\section{Donnan Exclusion Measurements}

The dialysis technique used was described recently. ${ }^{19} \mathrm{Na}^{+}$and $\mathrm{K}^{+}$ion concentrations inside and outside the dialysis bag were determined by atomic absorption spectroscopy (AAS), the $\mathrm{NO}_{3}{ }^{-}$ion concentrations were obtained by utilizing an anionic electrode (Orion) and the fractions of bound $\mathrm{Na}^{+}$and $\mathrm{K}^{+}$ions per carboxylate group, $\theta_{\mathrm{Na}}$ and $\theta_{\mathrm{K}}$, were computed by the procedure presented in ref 19 .

\section{The Dye Spectrophotometric Method}

$\mathrm{Mg}^{2+}$ concentrations were determined using a dye spectrophotometric method. Briefly, this method uses a weak dye-metal ion equilibrium as a concentration probe for the metal ion. By working at very low dye concentrations relative to those of the metal ion and polyion concentration the polyion-counterion binding is not disturbed by the dye.

Here, the dye is Eriochrome Black T (EBT) and the divalent counterion is $\mathrm{Mg}^{2+}$. The binding constant of the EBT- $\mathrm{Mg}^{2+}$ complex ${ }^{21}$ is of the order of 4000 at $\mathrm{pH}=8$ and ionic strength $0.1 \mathrm{M}$. Thus, the equilibrium $\mathrm{Mg}^{2+}+$ $\mathrm{EBT} \rightleftharpoons \mathrm{Mg}^{2+} \mathrm{EBT}$ is neither fully to the right nor to the left. The equilibrium constant depends, however, strongly on $\mathrm{pH}$. All solutions must be therefore carefully buffered. This was achieved using tris(hydroxymethyl)aminomethane as the buffer. This has a good buffering capacity at $\mathrm{pH}=8$ and low concentrations (up to $1 \times 10^{-3} \mathrm{M}$ ) can be used. In addition, the cation, tris(hydroxymethyl)-methylammonium, is fairly large. Thus, interferences in activity measurements of $\mathrm{Na}^{+}, \mathrm{K}^{+}$, or $\mathrm{Mg}^{2+}$ ions are not expected.

The measurements were performed with a Perkin-Elmer $550 \mathrm{SE} / \mathrm{UV} / \mathrm{VIS}$ spectrophotometer, working in the dual beam mode. The optical cells were of $1 \mathrm{~cm}$ in height. They 
were thermostated at $25 \pm 0.1^{\circ} \mathrm{C}$. The wavelengths used were $\lambda^{\prime}=615 \mathrm{~nm}$ and $\lambda^{\prime \prime}=560 \mathrm{~nm}$.

The dye $\left(c_{\mathrm{D}}=3 \times 10^{-5} \mathrm{M}\right)$ was added to both reference and polyelectrolyte solutions just before carrying out absorbance measurements. This was required to minimize problems due to the decomposition of ETB.

\section{Potentiometric Titration}

Potentiometric titrations were carried out with a digital $\mathrm{pH}$-meter Model 91 with a glass electrode E 50 (both WTW). Each solution $(20 \mathrm{ml})$ was titrated at $25^{\circ} \mathrm{C}$ under an argon atmosphere with $\mathrm{NaOH}$ or $\mathrm{KOH}$. Readings of $\mathrm{pH}$ were considered reliable if they were reproducible within $\pm 0.01 \mathrm{pH}$ unit before and after each titration.

The degree of ionization, $\alpha$, was determined by the relation

$$
\alpha=\left[C_{\mathrm{e}}+C_{\mathrm{H}^{+}}-K_{\mathrm{w}} / C_{\mathrm{H}^{+}}\right] / C_{\mathrm{P}},
$$

where $C_{\mathrm{e}}$ is the number of $\mathrm{NaOH}$ or $\mathrm{KOH}$ moles titrated, $C_{\mathrm{H}^{+}}$the $\mathrm{H}^{+}$-molarity and $K_{\mathrm{w}}$ the water constant. $C_{\mathrm{P}}$ is the concentration of the carboxylic acid group. It was calculated from the $\mathrm{NaOH}$ or $\mathrm{KOH}$-concentration at the end point.

Analysis was made in the usual manner. The $\mathrm{pH}$ of a poly(carboxylic acid) solution is related to the electrostatic free energy $\Delta G_{\mathrm{el}}(\alpha)$ by

$$
\mathrm{pH}=\mathrm{p} K_{0}-\log [(1-\alpha) / \alpha]+0.434 \Delta G_{\mathrm{el}}(\alpha) / k_{\mathrm{B}} T,
$$

where $\mathrm{p} K_{0}$ is the negative logarithm of the intrinsic dissociation constant of a carboxylic acid group which is defined as

$$
K_{0} \equiv \frac{C_{\mathrm{H}^{+}} C_{\mathrm{COO}^{-}}}{C_{\mathrm{COOH}}}
$$

Introducing the apparent logarithm ionization constant, $\mathrm{p} K_{\mathrm{a}}$, where

$$
\mathrm{p} K_{\mathrm{a}}(\alpha) \equiv \mathrm{pH}+\log [(1-\alpha) / \alpha],
$$

eq 3 can be rewritten in

$$
\mathrm{p} K_{\mathrm{a}}(\alpha)=\mathrm{p} K_{0}+0.434 \Delta G_{\mathrm{el}}(\alpha) / k_{\mathrm{B}} T
$$

so that

$$
G_{\mathrm{el}}(\alpha)=2.3 k_{\mathrm{B}} T \int_{0}^{\alpha}\left[\mathrm{p} K_{\mathrm{a}}(\alpha)-\mathrm{p} K_{0}\right] \mathrm{d} \alpha .
$$

$G_{\mathrm{el}}(\alpha)$ is the eletrostatic free energy per ionizable carboxylic group at a given degree of ionization, $\alpha$, i.e., $G_{\mathrm{el}}(\alpha)$ corresponds to the area under the curve $\mathrm{p} K_{\mathrm{a}}(\alpha)-\mathrm{p} K_{0}$ versus $\alpha$. Here, it is expected that $G_{\mathrm{el}}(\alpha)$ depends on the degree of hydrolysis.

\section{RESULTS AND DISCUSSION}

\section{Activity Coefficients}

There are two interesting theories for counterion activity coefficients. One was developed by Manning ${ }^{11}$ and the other, by Iwasa et $a l .^{12}$ According to Manning it holds:

if $\quad \xi<\xi_{c}$,

then

$$
\gamma_{+}=\exp (-0.5 \xi X /(X+2))
$$

and if $\xi \geq \xi_{\mathrm{c}}$

then

$\gamma_{+}=\frac{(X / \xi)+1}{X+1} \exp \left(-0.5 \xi^{-1} X /\left(\xi^{-1} X+2\right)\right)$

Here, $X$ is the ratio of polyelectrolyte equivalent concentration $C_{\mathrm{P}}$ to simple salt concentration $C_{\mathrm{s}}$, i.e., $X=C_{\mathrm{p}} / C_{\mathrm{s}}$. $\xi_{\mathrm{c}}$ is the critical charge density parameter. For univalent counterions $\xi_{\mathrm{c}}=1$.

The calculations of Iwasa et al. base on the cluster theory. Their results can be expressed as

$\xi<\xi_{\mathrm{c}}$

$\gamma_{+}=$

$$
\exp \left[\frac{\xi X}{X+2}\left(-0.5+0.3906 \xi\left(\frac{X}{X+2}-1\right)\right)\right]
$$




$$
\begin{aligned}
\xi \geq \xi_{\mathrm{c}} & \\
\gamma_{+} & =\frac{(X / \xi)+1}{X+1} \exp \left[\frac{\xi^{-1} X}{\xi^{-1} X+2}\right. \\
& \left.\times\left(-0.5+0.3096\left(\frac{\xi^{-1} X}{\xi^{-1} X+2}-1\right)\right)\right]
\end{aligned}
$$

Note, both formula sets are limiting laws and applicable only when solutions are dilute $\left(C_{\mathrm{P}}<0.1 \mathrm{M}\right)$.

To test these theories, sodium and potassium ion activity coefficients were measured in aqueous solutions of PAA and PAM- $y$ at $25^{\circ} \mathrm{C}$. Each polyelectrolyte was studied at three different concentrations $C_{\mathrm{p}}$ in the range of 0.005 to $0.015 \mathrm{M}$. The $\mathrm{NaNO}_{3}$ concentrations were varied so that $X$ ranged from 0.3 to 15 at each $C_{\mathbf{p}}$.

Activity coefficients must be corrected for mobile ion-mobile ion interactions before they can be compared to the limiting laws. The correction procedure used was, therefore, that employed by Wells ${ }^{22}$ and Kwak. ${ }^{23}$ It can be stated as,

$$
\gamma_{+}^{\mathrm{c}}=\gamma_{+}^{\exp } / \gamma_{+}^{0}
$$

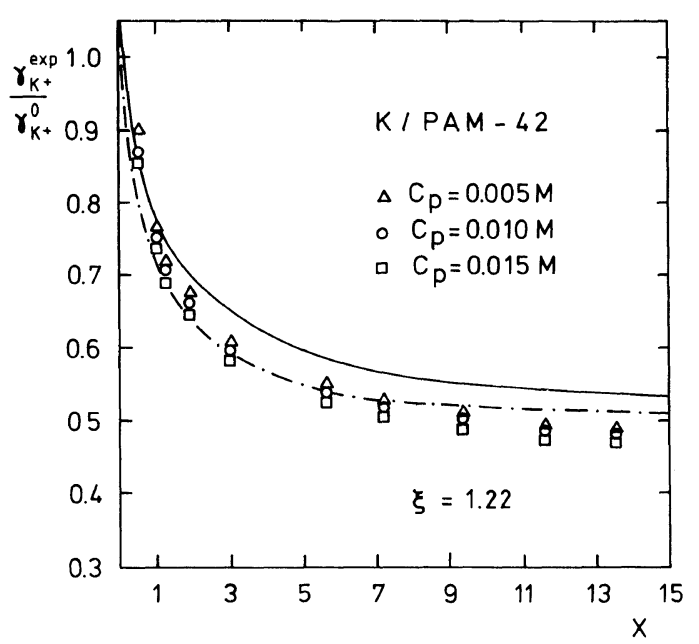

Figure 1. Dependence of $\gamma_{\mathrm{K}^{2}}^{\exp } / \gamma_{\mathbf{K}^{+}}^{0}$ on $X$ at various polyion concentrations in aqueous solutions of K-PAM-42 $(\xi=1.22)$. $C_{\mathbf{P}}=0.005(\triangle), 0.010(\bigcirc)$, and $0.015 \mathrm{M}(\square)$. Solid and dashed-dotted lines are calculated from eq 9 and 11 , respectively. $T$ is $25^{\circ} \mathrm{C}$. where $\gamma_{+}^{\exp }$ and $\gamma_{+}^{\mathrm{c}}$ are the experimental and corrected counterion activity coefficients. $\gamma_{+}^{0}$ is the activity coefficient of the added salt in the absence of polyelectrolyte. Values of $\gamma_{\mathrm{Na}^{+}}^{0}$ and $\gamma_{\mathbf{K}}^{0}$ + were obtained as $\gamma_{ \pm}^{0}$ values from Robinson and Stokes. ${ }^{24}$

For $\xi=1.22$ the activity coefficients measured are shown in Figure 1. The data are plots of $\gamma_{\mathrm{K}^{+}}^{\mathrm{c}}$ versus $X$. They do not show a significant $C_{\mathrm{P}}$-dependence for the concentration range studied. This is in accord with the above theories, which predict that $\gamma_{+}^{\mathrm{c}}$ is independent of $C_{\mathrm{P}}$. It is also in agreement with the experimental results of Kowblansky and Zema. $^{25}$

The dependence of the activity coefficients on the charge density parameter, $\xi$, is illustrated in Figures 2 and 3, where $\xi$ is larger than 1 in

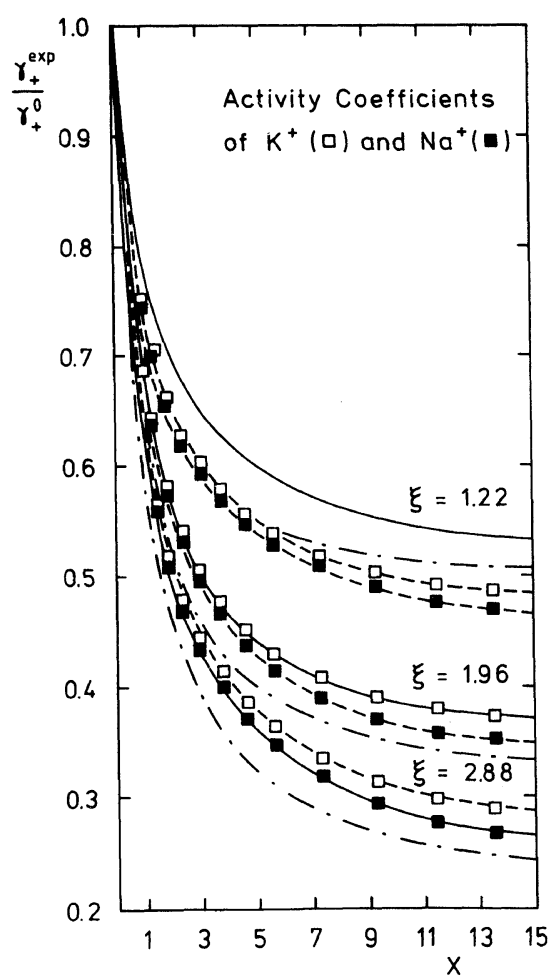

Figure 2. Corrected activity coefficients $\gamma_{+}^{\exp } / \gamma_{+}^{0}$ of $\mathrm{Na}^{+}$ and $\mathrm{K}^{+}$ions versus $X=C_{\mathrm{P}} / C_{\mathrm{S}}$ in aqueous solutions of PAA $(\xi=2.88)$, PAM-68 $(\xi=1.96)$, and PAM-42 $(\xi=1.22)$. Solid and dashed-dotted lines are from the Manning and Iwasa theories, respectively. $C_{\mathrm{P}}$ is $0.01 \mathrm{M}$ and $T$ is $25^{\circ} \mathrm{C}$. 
Figure 2 and smaller than 1 in Figure 3. Also shown are the Manning and Iwasa limiting curves.

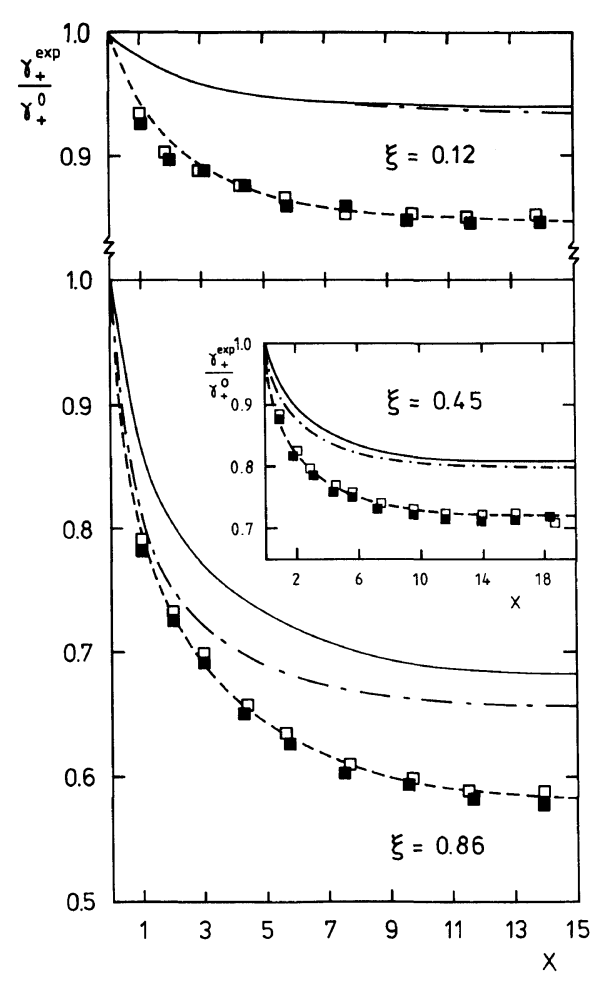

Figure 3. Corrected activity coefficients $\gamma_{+}^{\exp } / \gamma_{+}^{0}$ of $\mathrm{Na}^{+}$ and $\mathrm{K}^{+}$ions versus $X=C_{\mathrm{P}} / C_{\mathrm{S}}$ in aqueous solutions of PAM-30 $(\xi=0.86)$, PAM-16 $(\xi=0.45)$, and PAM-4 $(\xi=0.12) . C_{\mathrm{P}}$ is $0.01 \mathrm{M}$ and $T=25^{\circ} \mathrm{C}$. Symbols and lines as in Figure 2.
We see that the activity coefficients follow qualitatively the limiting laws, i.e., $\gamma_{\mathrm{Na}^{+}}^{\mathrm{c}}$ and $\gamma_{\mathrm{K}^{+}}^{\mathrm{c}}$ decrease with increasing $X$. At low $X$, the Manning and Iwasa predictions are not very different. For $\xi>1$, there is reasonably good agreement with both theories as long as $X<2$. As $X$ increases, there is disparity between experiment and theory. Then, $\gamma_{\mathbf{N a}^{+}}^{\mathrm{c}}$ is always significantly smaller than $\gamma_{\mathrm{K}^{+}}^{\mathrm{c}}$. This gives rise to the ion series $\mathrm{Na}^{+}>\mathrm{K}^{+}$.

If $\xi<1$, the downward shifts of $\gamma^{\mathrm{c}}+$ are much less than predicted by the theories. Additionally, the $\gamma_{\mathrm{Na}}^{\mathrm{c}}$ - values tend to be closer to the $\gamma_{\mathbf{K}^{+}}^{\mathrm{c}}$ values as for $\xi>1$. This point will be discussed later.

That is, for $\xi<1$, there is a significant discrepancy between experiment and theory. This is also found by Joshi and Kwak for sodium pectinates ${ }^{26}$ and by Kowblansky and $Z_{\text {Zema }}{ }^{25}$ for PAM- $y$. It was proposed by the latter that counterion condensation takes place possibly below $\xi_{c}=1$, as well as above. This may be right, as will be seen later.

The $\gamma_{\mathbf{N a}^{+}}^{\mathrm{c}}$ and $\gamma_{\mathbf{K}^{+}}^{\mathrm{c}}$ data can be well fitted by eq 8 to 9 if the parameter $\xi$ is chosen appropriately. The values of $\xi$ required to obtain a reasonable fit are listed in Table II. They are called $\xi_{\text {eff }}$, where the suffix "eff" stands for effective. For $\xi<1$ and also for $\xi>1$, $\xi_{\text {eff }}$ increases with decreasing $\xi$. This may have two reasons.

Table II. Microenvironmental effective dielectric constants and effective $\xi$-values calculated from the Manning theory

\begin{tabular}{|c|c|c|c|c|c|c|c|c|}
\hline \multirow{3}{*}{$\begin{array}{l}\text { Method } \\
\text { Ion } \\
\text { Sample }\end{array}$} & \multicolumn{4}{|c|}{ Activity measurement } & \multicolumn{4}{|c|}{ Counterion binding } \\
\hline & \multicolumn{2}{|c|}{$\mathrm{Na}^{+}$} & \multicolumn{2}{|c|}{$\mathrm{K}^{+}$} & \multicolumn{2}{|c|}{$\mathrm{Na}^{+}$} & \multicolumn{2}{|c|}{$\mathrm{K}^{+}$} \\
\hline & $\varepsilon_{\text {eff }}$ & $\xi_{\text {eff }}$ & $\varepsilon_{\text {eff }}$ & $\xi_{\text {eff }}$ & $\varepsilon_{\text {eff }}$ & $\xi_{\text {eff }}$ & $\varepsilon_{\text {eff }}$ & $\xi_{\text {eff }}$ \\
\hline PAM-4 & 25.2 & 0.37 & 25.2 & 0.37 & - & - & - & - \\
\hline PAM-10 & 38.2 & 0.58 & 38.2 & 0.58 & - & - & - & - \\
\hline PAM-16 & 45.8 & 0.75 & 45.8 & 0.75 & - & - & - & - \\
\hline PAM-30 & 60.1 & 1.12 & 60.1 & 1.12 & 58.5 & $1: 15$ & 58.5 & 1.15 \\
\hline PAM-42 & 67.5 & 1.42 & 68.9 & 1.39 & 66.1 & 1.45 & 66.5 & 1.44 \\
\hline PAM-68 & 73.4 & 2.08 & 78.3 & 1.95 & 74.9 & 2.04 & 78.5 & 1.96 \\
\hline PAA & 78.5 & 2.88 & 86.3 & 2.62 & 82.5 & 2.74 & 78.5 & 2.88 \\
\hline
\end{tabular}


With decreasing $\xi$, the average separation between neighboring polyion charges becomes larger. Thus, the repulsion between the charges may not be strong enough so that some folding of the polyion occurs. This would result in a larger effective value of $\xi$.

Note, folding is a conformational change within a single polymer chain. Probably, it is controlled via intramolecular hydrogen bonds. Intermacromolecular bonds can be excluded because light scattering measurements yield unchanged molar masses.

Experimental support for this mechanism can be obtained from studies on aqueous polyacrylamide solutions. There are several effects which indicate the existence of hydrogen bonds for PAM. For instance, there is a decrease in the intrinsic viscosity ${ }^{34}$ with time, while no viscosity loss could be observed when structure breakers ${ }^{35}$ such as formamide or propanol are added to the solutions. Other evidence are flocculation tests $^{36}$ and IR investigations $^{37}$ on deuterated and undeuterated PAM.

Viscosity decrease was also observed for PAM- $y^{38}$ and poly(L-glutamic acid), ${ }^{39}$ PGA. It is found that the viscosity loss of aqueous PAM- $y$ solutions is more drastic than of aqueous PAM solutions. Moreover, time dependent estimations of the $\alpha$-helix content of PGA from circular dichroism measurements show a decrease during the storage of the aqueous solutions from $53.3 \%$ (1st day) to $7.4 \%$ (26th day) at $25^{\circ} \mathrm{C}$. Unfortunately, there is, however, no clear evidence for $\alpha$-helix in aqueous PAM- $y$ solutions.

Therefore, the other possibility is that there is no folding but that the dielectric constant $\varepsilon_{\text {eff }}$ at the polyion surface is different from that of the solvent. Values of $\varepsilon_{\text {eff }}$ can be obtained from $\xi_{\text {eff }}$. They are listed in Table II. We see that $\varepsilon_{\text {eff }}$ decreases as $\xi$ or $y$ decreases. However, the dielectric constant of an amide group is larger than that of a carboxylate group. The number of amide groups per PAM- $y$ molecule increases with decreasing $y$.
That is, the dielectric constant, $\varepsilon_{\text {eff }}$, at the polyion surface should increase and not decrease. Thus, we have a dilemma.

If there is some folding, the $\mathrm{NH}_{2}$ and $\mathrm{COOH}$ groups are localized inside the helical segments, while the outside is built by the hydrophobic polyion backbone. We believe that the content of helical segments per PAM- $y$ molecule increases with decreasing $y$. Thus, a PAM-y becomes more hydrophobic as $y$ is lowered and the dielectric constant $\varepsilon_{\text {eff }}$ decreases.

Probably, both a change in $b$ and a change in $\varepsilon_{\text {eff }}$ are realized, where one effect may be dominant.

\section{Counterion Binding}

Figure 4 shows the binding isotherms for the systems $\mathrm{Na} / \mathrm{Mg} / \mathrm{PAA}$ and $\mathrm{K} / \mathrm{Mg} / \mathrm{PAA}$ at various ionic strengths in the range of $5 \times 10^{-3} \mathrm{M}$ to $1.5 \times 10^{-2} \mathrm{M}$. The graphs are plots of $\theta_{2}$ versus $C_{\mathrm{Mg}} / C_{\mathrm{P}}$, where $\theta_{2}$ is the fraction of bound

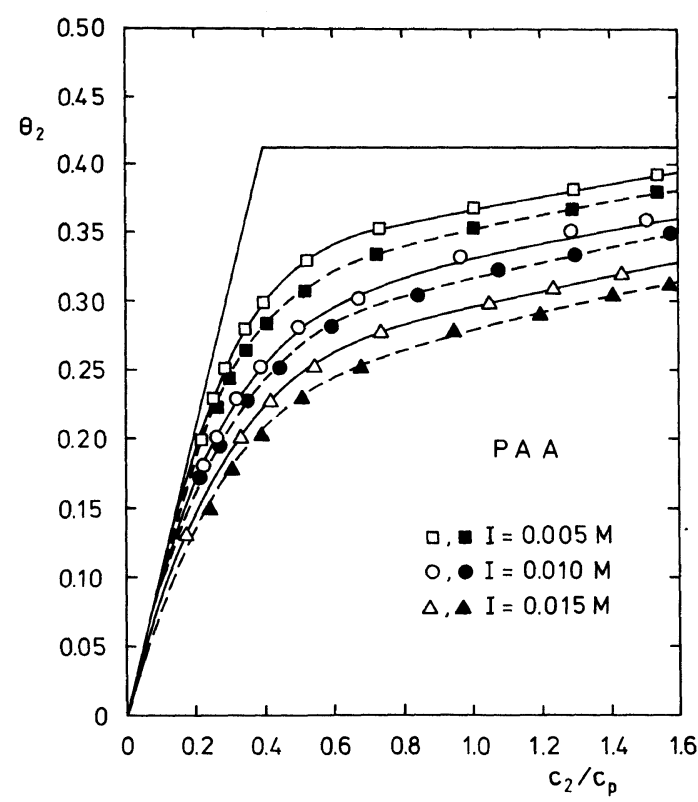

Figure 4. Plots of $\theta_{2} v s . C_{2} / C_{\mathrm{P}}$ for PAA at various ionic strengths, $I$. Open symbols are for potassium and the closed one for sodium. Upper line: simple condensation theory $(I=0)$. Lower curves: two-variable theory at different $I$. The parameter $\xi$ is 2.88 for the solid lines $\left(\mathrm{K}^{+}\right)$and 2.74 for the dashed lines $\left(\mathrm{Na}^{+}\right)$. 
divalent ions $\left(\mathrm{Mg}^{2+}\right)$ per carboxylate group. $C_{\mathrm{P}}$ is $0.0015 \mathrm{M}$ and $C_{\mathrm{Mg}}$ is the total $\mathrm{Mg}^{2+}$-ion concentration. Values of $\theta_{2}$ were determined via dye spectroscopy.

Also shown in Figure 4 are the theoretical curves of Manning's two-variable theory (solid lines). They were calculated by numerical iteration of eq 6 of ref 19. The discontinuous upper line was calculated from Manning's original condensation theory. This line will be reached when $\theta_{2}$ is extrapolated to $I=0 \mathrm{M}$.

The experimental isotherms show a smooth increase in $\theta_{2}$ with increasing $C_{\mathrm{Mg}} / C_{\mathrm{P}}$. This is in reasonable agreement with the two-variable theory. However, the $\theta_{2}(\mathrm{~K} / \mathrm{Mg} / \mathrm{PAA})$-values are slightly larger than the $\theta_{2}(\mathrm{Na} / \mathrm{Mg} / \mathrm{PAA})$ values at large $C_{\mathrm{Mg}} / C_{\mathrm{P}}$.

$\mathrm{Mg}^{2+}$ and $\mathrm{Na}^{+}$(or $\mathrm{K}^{+}$) ions are in competition for binding. $\mathrm{A} \mathrm{Mg}^{2+}$ ion can bind only when simultaneously two $\mathrm{Na}^{+}$(or $\mathrm{K}^{+}$) ions are released. Note, $\mathrm{Na}^{+}\left(\right.$or $\mathrm{K}^{+}$) ions are present in excess.

Thus, the isotherms of Figure 4 can be explained as follows. The $\mathrm{Mg}^{2+}$ ions are better able to displace $\mathrm{K}^{+}$than $\mathrm{Na}^{+}$ions from the region surrounding the PAA polyion. This leads to the specificity sequence $\mathrm{Na}^{+}>\mathrm{K}^{+}$, i.e., $\mathrm{Na}^{+}$is stronger bound by PAA than $\mathrm{K}^{+}$. Similar specificities were found for other carboxylate polyanions in the absence of divalent ions. For alginates Ander $^{27}$ found, for instance, from EMF measurements that $\gamma_{\mathbf{K}^{+}}^{\mathrm{c}}>\gamma_{\mathrm{Na}^{+}}^{\mathrm{c}}$. There are also authors who have arrived at no specificity and others ${ }^{28}$ who have deduced the reverse sequence $\mathrm{K}^{+}>\mathrm{Na}^{+}$. However, in most cases, where carboxylate polyanions were investigated, the affinity sequence $\mathrm{Li}^{+}>\mathrm{Na}^{+}>\mathrm{K}^{+}>\mathrm{Cs}^{+}$was found. That is, $\theta_{1}$, the fraction of bound univalent counterions per carboxylate group, decreases with decreasing hydrated radius of the cation.

Note, the same sequency ${ }^{29}$ is found for polyphosphates, while the reverse sequency holds for polysulfonates and polysulfates. Obviously, such an influence is not incorporated into the theory. That is, an

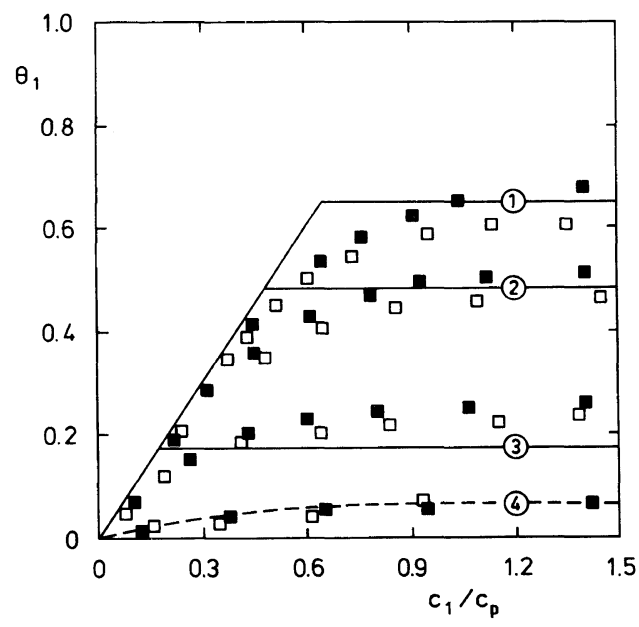

Figure 5. Plots of $\theta_{1}$ versus $C_{1} / C_{\mathrm{P}}$ for PAA (1), PAM-68 (2), PAM-42 (3), and PAM-30 (4). $C_{\mathrm{P}}$ is $0.001 \mathrm{M}$ and $T$ is $25^{\circ} \mathrm{C}$. (口) $\mathrm{Na}^{+}$and $(\square) \mathrm{K}^{+}$. Solid curves: simple condensation theory. Dashed curve: best fit for PAM-30.

explanation cannot be given yet.

We now turn our attention to the main topic of this paper, i.e., the influence of the charge density parameter, $\xi$, on the fraction of bound uni- and divalent counterions. The critical value of $\xi$ is 1 for univalent and $1 / 2$ for divalent counterions. Thus, four cases are interesting. They are $\xi \geq 1$ and $\xi<1$ for univalent cations and $\xi \geq 1 / 2$ and $\xi<1 / 2$ for divalent cations.

Figure 5 shows isotherms for the systems Na/PAM- $y$ and K/PAM- $y$. $\xi$ is larger than 1 and no divalent cations are present. The graphs are plots of $\theta_{1}$ versus $C_{1} / C_{\mathrm{P}}$, where $\theta_{1}$ is the fraction of bound univalent counterions. Values of $\theta_{1}$ were determined by the dialysis method. $C_{1}$ is the total concentration of the $\mathrm{Na}^{+}$(or $\mathrm{K}^{+}$) ions at dialysis equilibrium and $C_{\mathrm{P}}$ was $0.001 \mathrm{M}$. Also shown are the theoretical isotherms of the simple condensation model. Obviously, the agreement between theory and experiment is sufficient. For a given value of $C_{1} / C_{\mathrm{P}}, \theta_{1}$ decreases with decreasing $\xi$. Simultaneously, the deviations between $\theta_{\mathrm{Na}^{+}}$ and $\theta_{\mathrm{K}^{+}}$become smaller. Both effects are in line with the measurements of the activity coefficients. 
For $\xi<1$, theory predicts $\theta_{1}=0$. Experimentally, the situation is somewhat diffuse. The values obtained for $\theta_{1}$ by the dialysis method are of the order of zero $\left(\theta_{1}<0.05\right)$, but they are affected with errors of 20 and more $\%$. Thus, one could conclude that $\theta_{1}=0$. However, we have also performed some measurements by Na-NMR and ESR. It is found that $\theta_{1}$ is small, but larger than zero within the experimental error of $5-10 \%$. That is, it seems that univalent counterions can condense for $\xi \leq \xi_{\mathrm{c}}$, as well as for $\xi>\xi_{\mathrm{c}}$. The reason why counterion condensation occurs even for $\xi \leq \xi_{\mathrm{c}}$ may be folding. On the other hand, it can not excluded completely that condensation takes place if there is no folding. To prove this, it is desirable to extend the studies for $\xi \leq \xi_{\mathrm{c}}$ to other types of polyions and to other univalent counterions. Such experiments are now in progress.

Figure 6 presents isotherms for the systems

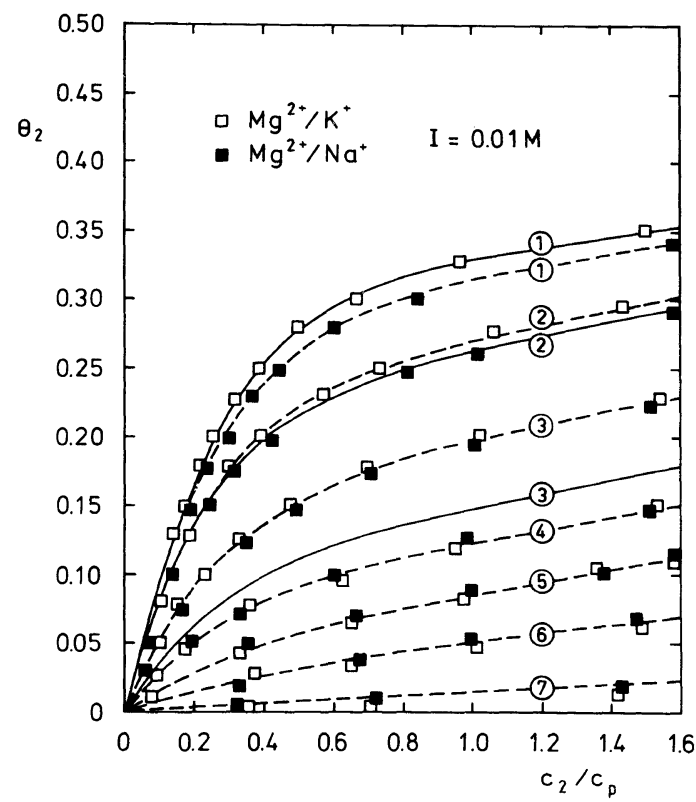

Figure 6. Binding isotherms of $\theta_{2}$ versus $C_{2} / C_{\mathrm{P}}$ for the systems $\mathrm{Mg} / \mathrm{Na}(\mathrm{K}) / \mathrm{PAA}$ and $\mathrm{Mg} / \mathrm{Na}(\mathrm{K}) / \mathrm{PAM}-y . C_{\mathrm{P}}$ is $0.0015 \mathrm{M}, I$ is $0.01 \mathrm{M}$ and $T$ is $25^{\circ} \mathrm{C}$. (1) $\xi=2.88$; (2) $\xi=1.96$; (3) $\xi=1.22$; (4) $\xi=0.86$; (5) $\xi=0.45$; (6) $\xi=0.28$; (7) $\xi=0.12$. Solid lines: two-variable theory for $\xi$. Dashed lines: two-variable theory for $\xi_{\text {eff }}$.
$\mathrm{Na} / \mathrm{Mg} / \mathrm{PAM}-y$ and $\mathrm{K} / \mathrm{Mg} / \mathrm{PAM}-y . \quad C_{\mathrm{P}}$ is $0.0015 \mathrm{M}$ and $I=0.01 \mathrm{M}$. Included are also the theoretical curves of the two-parameter theory. Four effects can be observed.

First, $\theta_{2}$ decreases with decreasing $\xi$ for a given ratio of $C_{2} / C_{\mathrm{P}}$. This confirms that binding is strongly dependent on $\xi$, i.e., on the average separation of polyion charges, and not on the exact polyion structure.

Second, the experimental $\theta_{2}$-values become considerably larger than the theoretical values when $\xi$ decreases. Similar observations were made by $\mathrm{Kohn}^{30}$ for the interactions of $\mathrm{Ca}^{2+}$ and $\mathrm{Sr}^{2+}$ ions with polygalacturonate in the absence of added univalent salts. Kohn has explained the high degree of $\mathrm{Ca}^{2+}$ (or $\mathrm{Sr}^{2+}$ )-binding as intermolecular binding of $\mathrm{Ca}^{2+}\left(\right.$ or $\mathrm{Sr}^{2+}$ ) ions with carboxylate groups of different chains in small aggregates. This may be also the case here, but it is also possible that $\mathrm{Mg}^{2+}$ ions interact with PAM-y in a specific type of interaction, i.e., by site-binding formation. $\theta_{2}$ would then be the sum of two terms, i.e., $\theta_{2}$ would be $\theta_{2, \mathrm{t}}+\theta_{2, \mathrm{~s}}$, where $\theta_{2, \mathrm{t}}$ and $\theta_{2, \mathrm{~s}}$ are the fractions of the divalent counterions that are bound territorial and by site-binding. The third possibility is again that polyion folding occurs, so that $\xi$ should be replaced by $\xi_{\text {eff }}$. Values obtained for $\xi_{\text {eff }}$ by taking $\xi$ as an adjustable parameter are shown in Table II. They are in reasonable agreement with the $\xi_{\text {eff }}$-values deduced from the activity measurements.

Third, the disparity between $\theta_{2}\left(\mathrm{Na}^{+}\right)$and $\theta_{2}\left(\mathrm{~K}^{+}\right)$vanishes as $\xi$ becomes smaller than 1 . This may indicate that $\theta_{1}$ is zero for $\xi<1$ as predicted by theory. Alternatively, it is thinkable that the affinity of binding is equal for $\mathrm{Na}^{+}$and $\mathrm{K}^{+}$at $\xi<1$.

Fourth, $\theta_{2}$ is not zero for $\xi<0.5$ within the experiment errors. This is contrary to the theory, which predicts that for divalent ions $\xi_{c}=0.5$. However, it would be strange, if univalent counterions could condense for $\xi \leq \xi_{\mathrm{c}}$, while divalent counterions could not. The explanation is the same as be before. 
Folding may occur. This would produce polyion parts (segments), possessing $\xi$-values which are larger than 0.5 . The other possibility is that the dielectric constant $\varepsilon_{\text {eff }}$ at the polyion surface is much smaller than that of water. Probably, both effects act simultaneously.

\section{pH-Induced Conformational Transitions}

A good method to detect conformational transitions such as folding is the potentiometric titration. The titration curves of polyacids which undergo a conformational transition greatly differ from those of polyacids without transition. A discontinuous change of $\mathrm{p} K_{\mathrm{a}}$ versus the degree of ionization, $\alpha$, is observed in the former case while a gradual increase of $\mathrm{p} K_{\mathrm{a}}$ is observed in the absence of any special transition. For instance, poly(methylacryl acid), PMAA, and poly(L-lysine co 1.3 benzenedisulphonyl chloride), PLL, show such features. ${ }^{31,32}$

Figure 7 shows plots of $\mathrm{p} K_{\mathrm{a}}$ versus $\alpha$ for Na-PAA, K-PAA, Na-PAM- $y$, and K-PAM- $y$ having various degrees of hydrolysis. The solutions contained no added salt. $C_{\mathrm{P}}$ was $0.0012 \mathrm{M}$.

There is a clear difference in behaviour

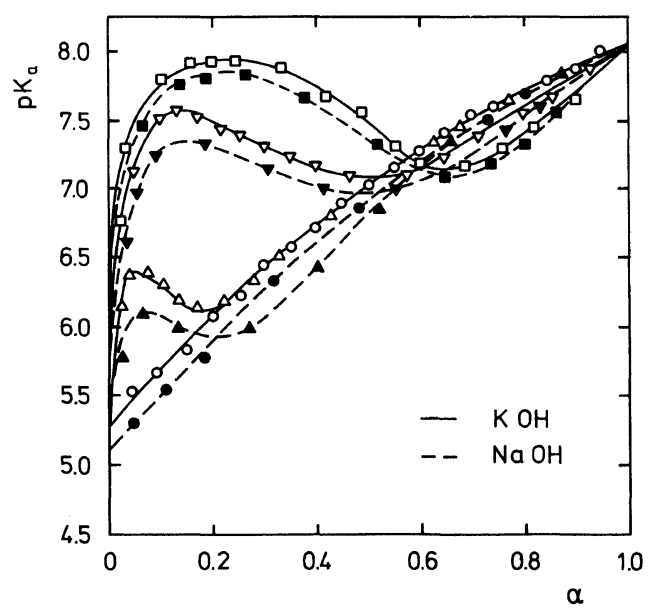

Figure 7. $\mathrm{p} K_{\mathrm{a}}$ values of PAA and PAM- $y$ neutralized by $\mathrm{KOH}$ and $\mathrm{NaOH}$ in the absence of added salt. $C_{\mathrm{P}}$ is $0.012 \mathrm{M}$ and $T$ is $25^{\circ} \mathrm{C} . y=100 \%(\bigcirc, \bigcirc) ; 68 \%(\triangle, \Delta) ; 42 \%(\nabla$, $\nabla) ; 30 \%(\square, \square)$. Open symbols: $\mathrm{KOH}$. Closed symbols: $\mathrm{NaOH}$. between PAA and PAM- $y$. For PAA, $\mathrm{p} K_{\mathrm{a}}$ is a monotonic increasing function of $\alpha$, while for PAM- $y$ there is an abnormal rise of $\mathrm{p} K_{\mathrm{a}}$ at low degrees of ionization. This abnormality of $\mathrm{p} K_{\mathrm{a}}$ is due to the intramolecular association of PAM- $y$ segments via hydrogen binding. It is often called the globular-coil transition, although it may be a helix-coil transition (see, for instance, ref 35-40).

At small $\alpha, \mathrm{p} K_{\mathrm{a}}$ of PAM- $y$ increases with decreasing degree of hydrolysis $y$. Additionally, the transition region is shifted to higher values of $\alpha$ as the number of amide groups per polyion increases.

Very similar curves are obtained with added salt. It is observed that the curves of PAA and PAM-y become lower with increasing ionic strength. Any discussion of this effect is, however, beside the purpose of this paper. It is of more interest whether the number of amide groups around an carboxylate group can change the selectivity for counterion binding. $\mathrm{p} K_{\mathrm{a}}$-curves of K-PAA and K-PAA- $y$ are always higher than those of the Na-polyions. That is, the electrostatic energy $G_{\mathrm{el}}(\alpha)$ required to remove $\mathrm{H}^{+}$from PAA or PAM- $y$ is greater when titration is performed with $\mathrm{KOH}$ than with $\mathrm{NaOH}$. It is thus more easy to replace $\mathrm{H}^{+}$ by $\mathrm{Na}^{+}$than by $\mathrm{K}^{+}$. That is, the affinity sequence is again $\mathrm{Na}^{+}>\mathrm{K}^{+}$.

The differences between $\mathrm{p} K_{\mathrm{a}}(\mathrm{Na})$ and $\mathrm{p} K_{\mathrm{a}}(\mathrm{K})$ become smaller with decreasing $\xi$. For $\xi<\xi_{\mathrm{c}}$, $\mathrm{p} K_{\mathrm{a}}(\mathrm{Na}) \approx \mathrm{p} K_{\mathrm{a}}(\mathrm{K})$. This is in agreement with the activity and binding measurements. That is, folding or something like folding is one factor in producing ion selectivity. The other factor is the type of the ionizable group such as $\mathrm{COO}^{-}, \mathrm{SO}_{3}{ }^{-}$or $\mathrm{PO}_{3}{ }^{2-}$. For $\mathrm{COO}^{-}$and $\mathrm{PO}_{3}{ }^{2-}$ we find $\mathrm{Na}^{+} \geq \mathrm{K}^{+}$and for $\mathrm{SO}_{3}{ }^{-}$we have $\mathrm{K}^{+} \leq \mathrm{Na}^{+}$.

The main result is that PAM-y shows a globular-coil transition. Folding is one reason for deviation observed between theory and experiment. 


\section{CONCLUSIONS}

The following conclusions can be drawn:

(1) For $\xi>1$, Manning's counterion condensation concept is sufficient to describe correctly the interactions between small ions and polyions. The agreement between theory and experiment is quite well for $\gamma_{+}^{\mathrm{c}}, \theta_{1}$, and $\theta_{2}$. This is remarkable because the Manning theory contains no adjustable parameter. The only defect is that the theory does not incorporate specific interactions.

(2) For $\xi<1$, there is significant deviation between theory and experiment. This holds for the activity coefficients and also for the fractions of counterion binding. The probable reasons are: (a) There is some polyion folding so that $\xi_{\text {eff }}$ is larger than $\xi$. This idea was manifested by potentiometric titration. The $\mathrm{p} K_{\mathrm{a}}$-values of PAM- $y$ show a curvature which is characteristical for a globular-coil transition. (b) The dielectric constant $\varepsilon_{\text {eff }}$ at the polyion surface is smaller than that of water. The provement of this idea is difficult. This is because $\varepsilon_{\text {eff }}$ can be measured only indirectly. This was done by Morishima et al. ${ }^{33}$ who estimated $\varepsilon_{\text {eff }}$ by linking covalently $\mathrm{pH}-$ sensitive chromophores to the polyelectrolyte molecular surface. Indeed the values obtained for $\varepsilon_{\text {eff }}$ are smaller than that of water $\left(\varepsilon_{\text {eff }}<80\right)$. However, it is not clear to what extent the chromophores influence the polyelectrolyte conformation.

(3) The question whether counterion condensation takes place for univalent ions below $\xi<1$ and for divalent ions below $\xi<0.5$ is still an unsolved problem. According to Ander ${ }^{10}$ there is a critical $\xi$ but according to Kowblansky and Zema ${ }^{25}$ there is none. Our results do not show clearly that $\xi_{c}$ exists or that $\xi_{\mathrm{c}}$ does not exist. Thus, the situation remains nebulous.

To bring light into this topic, effects such as folding or segment association should be excluded. One way to achieve this is to replace water by an organic solvent. This should lead to a decrease, and even disappearance, of the compact conformation if hydrophobic interactions are destroyed. An example is PMAA. In $50 \%$ methanol-water mixtures, there is no longer a discontinuity in $\mathrm{p} K_{\mathrm{a}}$.

There is a second problem. Generally, polyions with $\xi<1$ do not posses the conformation of a rod as assumed by Manning. That is, the solubility of a polyion in an organic solvent must be high, so that the polyion coil is highly expanded. Unfortunately, this is difficult to achieve. Therefore, it may be better to repeat Manning's calculations for molecular structures such as spheres, ellipsoids or wormlike chains. Perhaps, there are also critical charge density parameters.

To clarify these points, further study is in progress.

Acknowledgements. I thank Prof. Dr. M. D. Lechner for provision of facilities and S. Warm and B. Ahrens for excellent assistance. The research was supported by the BMFT and the Fonds der Chemischen Industrie.

\section{REFERENCES}

1. F. E. Harris and S. A. Rice, J. Phys. Chem., 58, 725 (1954).

2. S. A. Rice and M. Nagasawa, in "Polyelectrolyte Solutions," Academic Press, New York, N.Y., 1966.

3. J. S. Tan and S. P. Gasper, J. Polym. Sci., Polym. Phys. Ed., 13, 1705 (1975).

4. G. S. Manning, J. Chem. Phys., 51, 924 (1969).

5. Y. M. Joshi and J. C. T. Kwak, Biophys. Chem., 13, 65 (1981).

6. S. Koda, H. Nomura, and M. Nagasawa, Biophys. Chem., 23, 147 (1985).

7. T. Kanaya, K. Kaji, R. Kitamaru, B. Gabrys, and J. S. Higgins, J. Chem. Soc., Faraday Trans. 1, 84, 3487 (1988).

8. M. Kowblansky and P. Zema, Macromolecules, 14, 1448 (1981).

9. M. Kowblansky and P. Zema, Macromolecules, 15, 788 (1982).

10. P. Ander and M. Kardan, Macromolecules, 17, 2431 (1984).

11. G. S. Manning, in "Polyelectrolytes," E. Selegny, Ed., Reidel Publishing Co., Dordrecht, Holland, 1974. 
12. K. Iwasa and J. C. T. Kwak, J. Phys. Chem., 81, 408 (1977).

13. B. H. Zimm and M. LeBret, J. Biomol. Struct. Dyn., 1, 461 (1983).

14. C. Anderson, M. Record, and P. Hart, Biophys. Chem., 7, 301 (1978).

15. R. Trifiletti and P. Ander, Macromolecules, 12, 1197 (1979).

16. J. Klein and R. Heitzmann, Makromol. Chem., 179, 1895 (1978).

17. W. M. Kulicke and R. Kniewske, Makromol. Chem., 182, 2277 (1981).

18. H. Noguchi, K. Gekko, and S. Makino, Macromolecules, 6, 438 (1973).

19. E. Nordmeier and W. Dauwe, Polym. J., 23, 1297 (1991).

20. J. C. T. Kwak and Y. M. Joshi, Biophys. Chem., 13, 55 (1981).

21. H. Diehl and F. Lindstrom, Anal. Chem., 31, 414 (1959).

22. J. D. Wells, Proc. R. Soc. London, Ser. B., 183, 399 (1973).

23. J. C. T. Kwak, J.Phys. Chem., 77, 2790 (1973).

24. R. A. Robinson and R. H. Stokes, "Electrolyte Solutions," Butterworth's, London, 1970.

25. M. Kowblansky and P. Zema, Macromolecules, 14, 166 (1981).

26. Y. M. Joshi and J. C. T. Kwak, J. Phys. Chem., 83,
1978 (1979).

27. T. J. Podlos and P. Ander, Macromolecules, 3, 154 (1970)

28. N. Ise and T. Okubo, Macromolecules, 11, 439 (1978)

29. U. Strauß, in "Polyelectrolytes," E. Selegny, Ed., Reidel Publishing Co., Dordrecht, Holland, 1974.

30. R. Kohn, Pure Appl. Chem., 42, 371 (1975).

31. C. Braud and E. Selegny, J. Polym. Sci., 12, 2767 (1974).

32. J. C. Fenyo and E. Selegny, J. Polym. Sci., 12, 2659 (1974).

33. Y. Morishima, T. Kobayashi, and S. Nozakura, Macromolecules, 21, 101 (1988).

34. K. L. Gardner, W. R. Murphy, and T. G. Geehan, J. Appl. Polym. Sci., 22, 881 (1978).

35. W. M. Kulicke and R. Kniewske, Makromol. Chem., 181, 823 (1980).

36. W. M. Kulicke, R. Kniewske, and H. H. Hörl, Angew. Makromol. Chem., 87, 195 (1980).

37. W. M. Kulicke and H. W. Siesler, J. Polym. Sci., 20, 553 (1982).

38. W. M. Kulicke and R. Kniewske, Makromol. Chem., 182, 2277 (1981).

39. Ch. S. Cho, A. Nakagani, T. Komoto, and T. Kawai, Makromol. Chem., 179, 1345 (1978).

40. M. K. Gupta and R. Bansil, J. Polym. Sci., Phys. $E d .$, 19, 353 (1981). 